\title{
ON THE DIVISIBILITY GRAPH FOR FINITE SETS OF POSITIVE INTEGERS
}

\author{
ADELEH ABDOLGHAFOURIAN AND MOHAMMAD A. IRANMANESH
}

\begin{abstract}
Let $X$ be a finite set of positive integers. The divisibility graph $\mathscr{D}(X)$ is a directed graph with vertex set $X \backslash\{1\}$ and an arc from $a$ to $b$ whenever $a$ divides $b$. Since the divisibility graph and its underlying graph have the same number of connected components, we consider the underlying graph of $\mathscr{D}(X)$, and we denote it by $\mathrm{D}(X)$. In this paper, we will find some graph theoretical parameters of $\mathrm{D}(X)$, some relations between the structure of $\mathrm{D}(X)$, and the structure of known graphs $\Gamma(X), \Delta(X)$ and $B(X)$ will be considered. In addition, we investigate some properties of $\mathrm{D}(X Y)$ for the product of two non-empty subsets $X$ and $Y$ of positive integers.
\end{abstract}

\section{Introduction.}

1.1. Background and motivation. There are several graphs associated to a set of positive integers. Some of these graphs, such as the prime vertex graph and the common divisor graph, were introduced by Lewis [6]. Inspired by the connection between these two graphs, Praeger and the second author defined the bipartite divisor graph. In [2], the divisibility graph $\mathscr{D}(X)$ for a non-empty subset $X$ of positive integers was introduced. In [2, Question 7], the authors asked for the number of connected components of $\mathscr{D}(G)$ where $\mathscr{D}(G)=\mathscr{D}(\operatorname{cs}(G))$ and $\operatorname{cs}(G)$ is the set of conjugacy class sizes of a finite group $G$. As a partial answer to that question the authors [1] calculated the number of connected components of $\mathscr{D}(G)$, where $G$ is a symmetric or an alternating permutation group.

Let $X$ be a non-empty set of integers, and let $X^{*}=X \backslash\{1\}$. Since the number of connected components of $\mathscr{D}(X)$ and its underlying graph

2010 AMS Mathematics subject classification. Primary 05C25.

Keywords and phrases. Divisibility graph, bipartite graph, prime vertex graph, common divisor graph, connected component.

The second author is the corresponding author.

Received by the editors on January 13, 2015. 
is the same, we prefer to investigate the combinatorial properties of the underlying graph.

Our aim in this paper is to investigate some combinatorial properties of $\mathrm{D}(X)$ (in particular, the number of connected components) and find the relation between this graph and three important graphs, namely, $\Gamma(X), \Delta(X)$ and $B(X)$. For more information about $\Gamma(X), \Delta(X)$ and $B(X)$, we refer the reader to $[\mathbf{5}, \mathbf{6}]$. In addition, a survey on results for $B(X)$ and $B(G)$ can be found in [4].

In the following, we recall some important graphs associated with an arbitrary non-empty subset $X$ of positive integers (see $[\mathbf{2}, \mathbf{5}, \mathbf{6}]$ ).

(1) The common divisor graph $\Gamma(X)$ is a simple graph with vertex set $X^{*}$. Two elements of $X^{*}$ are adjacent whenever their greatest common divisor is greater than 1 .

(2) The prime vertex graph $\Delta(X)$ is a simple graph with vertex set $\rho(X)$. Two primes $p$ and $q$ are adjacent if there exist $x \in X$ such that $p q$ divides $x$.

(3) The bipartite divisor graph $B(X)$ is a bipartite graph with a vertex set which is a disjoint union of two sets $X^{*}$ and $\rho(X)$. The prime $p$ is adjacent to $x$ if and only if $p$ divides $x$.

(4) The divisibility graph $\mathscr{D}(X)$ is a directed graph with vertex set $X^{*}$. For two elements $a, b \in X^{*}$, there is a directed edge from $a$ to $b$ if $a$ divides $b$.

It can easily be seen that, if there exists a simple directed path between two distinct elements $a, b$ of $X^{*}$ in $\mathscr{D}(X)$, then $a$ divides $b$. This implies that there is no directed path from $b$ to $a$. Hence, to study the parameters of $\mathscr{D}(X)$, we consider its underlying graph which is denoted by $\mathrm{D}(X)$. Note that $\mathrm{D}(X)$ is a simple graph with vertex set $X^{*}$ and $E(\mathrm{D}(X))=\{\{a, b\} ; a$ divides $b$ or $b$ divides $a\}$.

1.2. Notation and statement of results. Throughout this paper, we consider simple graphs, where by a simple graph we mean a graph with no multiple edges or loops. Let $X$ be a finite set of positive integers and $X^{*}=X \backslash\{1\}$. For an arbitrary set $x \in X$,

$$
\rho(x)=\{p ; p \text { is prime and } p \text { divides } x\}
$$


and

$$
\rho(X)=\bigcup_{x \in X} \rho(x) .
$$

Let $\mathcal{G}=(V(\mathcal{G}), E(\mathcal{G}))$ and $\mathcal{H}=(V(\mathcal{H}), E(\mathcal{H}))$ be two simple graphs. We say $\mathcal{G}$ is isomorphic to $\mathcal{H}$, and we write $\mathcal{G} \cong \mathcal{H}$ if and only if there is a bijection $f: V(\mathcal{G}) \rightarrow V(\mathcal{H})$ such that $\{a, b\} \in E(\mathcal{G})$ whenever $\{f(a), f(b)\} \in E(\mathcal{H})$. For a vertex $v \in \mathcal{G}$, the set of its neighbors is denoted by $N(v)$. Moreover, if $S \subseteq V(\mathcal{G})$, the neighborhood of $S$ is defined by

$$
N(S)=\bigcup_{v \in S} N(v)
$$

A path and a cycle with $n$ vertices are denoted by $P_{n}$ and $C_{n}$, respectively. Let $\mathcal{G}$ be a graph. The distance between two vertices $a$ and $b$ in $\mathcal{G}$ is denoted by $d_{\mathcal{G}}(a, b)$, and, if the graph is clear from the context, we suppress $\mathcal{G}$ in our notation and write $d(a, b)$. For a graph $\mathcal{G}$, the diameter, number of connected components, connected components containing $x$ and the girth are denoted by $\operatorname{diam}(\mathcal{G}), n(\mathcal{G})$, $[x]_{\mathcal{G}}$ and $\mathrm{g}(\mathcal{G})$, respectively. For the definition of these parameters we refer the reader to [7]. Remember that, for an acyclic graph $\mathcal{G}$, the girth is 0 . For a disconnected $\operatorname{graph} \mathcal{G}$ we let $\operatorname{diam}(\mathcal{G})=\infty$.

In a simple directed graph $\mathscr{G}$ we denote the number of directed edges from $v$ to another vertex of $V(\mathscr{G})$ by $d^{+}(v)$. Also, $d^{-}(v)$ is the number of edges directed from another vertex to $v$. If there is a vertex $v$ such that $d^{-}(v)=0,\left(d^{+}(v)=0\right)$, we say that $v$ is a source $(\operatorname{sink})$. In addition,

$$
N^{+}(v)=\{w \in V(\mathscr{G}) ;(v, w) \in E(\mathscr{G})\}
$$

and

$$
N^{-}(v)=\{w \in V(\mathscr{G}) ;(w, v) \in E(\mathscr{G})\} .
$$

Other notation is common and may be found in [7].

In Section 2, we prove that a graph $\mathcal{G}$ is isomorphic to $\mathrm{D}(X)$ if and only if $\mathcal{G}$ is a comparability graph. In Section 3 , we investigate the relations among $\Delta(X), \Gamma(X), B(X)$ and $\mathrm{D}(X)$. In particular, we prove that, if any vertices of $B(X)$ have degree $<3$, then $\mathrm{D}(X)$ is acyclic. Finally, in Section 4, we find some conditions of two non-empty subsets 
$X$ and $Y$ so that $\mathrm{D}(X Y)$ is isomorphic to the strong product of $\mathrm{D}(X)$ and $\mathrm{D}(Y)$.

2. Representing graphs as $\mathrm{D}(X)$. In this section, we find some conditions for a simple graph to be the underlying graph of a divisibility graph.

A transitive orientation of a graph $\mathcal{G}$ is an orientation $\mathcal{D}$ such that, whenever $(x, y)$ and $(y, z)$ are edges in $\mathcal{D}$, there is also an edge $(x, z)$ in $\mathcal{G}$ that is oriented from $x$ to $z$ in $\mathcal{D}$. A simple graph $\mathcal{G}$ is a comparability graph if it has a transitive orientation (see [7, Definition 5.3.23]).

If $\mathcal{G}$ is a comparability graph, then we use $\overrightarrow{\mathcal{G}}$ to denote a directed graph obtained by its transitive orientation from $\mathcal{G}$. Note that a finite comparability graph may have more than one transitive orientation.

We claim that every finite comparability graph has at least one source. Conversely, suppose that $d^{-}(v)>0$ for every $v \in V(\overrightarrow{\mathcal{G}})$. Let $v_{1}$ be an arbitrary vertex and $\left(v_{2}, v_{1}\right) \in E(\overrightarrow{\mathcal{G}})$. Since $d^{-}(v)>0$ for every vertex, we choose a directed walk $W$ with end vertex $v_{1}$. Now the finiteness of $\mathcal{G}$ yields that $W$ contains a directed cycle $v_{i_{1}} v_{i_{2}} v_{i_{3}} \cdots v_{i_{k-1}} v_{i_{1}}$. Since the orientation on $\overrightarrow{\mathcal{G}}$ is transitive, it is possible to remove some vertices from this path and find that $\left\{v_{i_{1}} v_{i_{k}}\right\} \in E(\mathcal{G})$. Since $\left\{v_{i_{k}} v_{i_{1}}\right\}$ is an edge, this implies that $\mathcal{G}$ is not a simple graph, which is a contradiction.

In the following theorem, we show that any finite comparability graph is isomorphic to a divisibility graph $\mathrm{D}(X)$ for some finite set $X$.

Theorem 2.1. There exists a finite set $X \subseteq \mathbb{N}$ such that $\mathcal{G} \cong \mathrm{D}(X)$ if and only if the graph $\mathcal{G}$ is a comparability graph.

Proof. First, suppose that $\mathcal{G}$ is a comparability graph. Suppose that

$$
s=\max \left\{d^{-}(v) ; v \in V(\overrightarrow{\mathcal{G}})\right\} .
$$

Let

$$
N_{i}=\left\{v \in V(\overrightarrow{\mathcal{G}}) ; d^{-}(v)=i\right\},
$$

where $0 \leq i \leq s$. In particular, $N_{0}$ is the set of sources. 
Suppose that $v \in N_{i}$ and $w \in N_{j}$ where $i \leq j$. If $(w, v) \in E(\overrightarrow{\mathcal{G}})$, then transitivity of the orientation yields that $(x, v) \in E(\overrightarrow{\mathcal{G}})$ for every $x \in N^{-}(w)$. This means that $d^{-}(v) \geq d^{-}(w)+1$, which is a contradiction with $i \leq j$. Therefore,

$$
N^{-}\left(N_{i}\right) \subseteq \bigcup_{r=1}^{i-1} N_{r}
$$

for every $1 \leq i \leq s$. In particular, each $N_{i}$ is an independent set of $G$ for every $0 \leq i \leq s$.

For every $0 \leq i \leq s$, suppose that

$$
\left|N_{i}\right|=k_{i} \quad \text { and } \quad N_{i}=\left\{v_{i 1}, \ldots, v_{i k_{i}}\right\} .
$$

Suppose that

$$
\mathcal{P}_{i}=\left\{p_{i 1}, \ldots, p_{i k_{i}}\right\} \subseteq \mathbb{N}
$$

is a set of pairwise distinct primes such that $\mathcal{P}_{i} \cap \mathcal{P}_{j}=\emptyset$ where $i \neq j$. Let $N_{0}^{\prime}=\mathcal{P}_{0}$, and define $f_{0}: N_{0} \rightarrow N_{0}^{\prime}$ by

$$
f_{0}\left(v_{0 i}\right)=p_{0 i}, \quad 1 \leq i \leq k_{0} .
$$

For $1 \leq l \leq k$, let

$$
N_{l}^{\prime}=\left\{x_{l i} ; x_{l i}=p_{l i} \prod_{j=1}^{l} f_{l-1}\left(v_{(l-1) j}\right), 1 \leq i \leq k_{l}, v_{(l-1) j} \in N^{-}\left(v_{l i}\right)\right\},
$$

and let $f_{l}: N_{l} \rightarrow N_{l}^{\prime}$ be given by $f_{l}\left(v_{l t}\right)=x_{l t}$, where $1 \leq t \leq k_{l}$. Set

$$
X=\bigcup_{l=0}^{k} N_{l}^{\prime}
$$

and define $f: V(\overrightarrow{\mathcal{G}}) \rightarrow X$ by $\left.f\right|_{N_{l}}=f_{l}$. Then, $f$ is a bijection from $V(\overrightarrow{\mathcal{G}})$ to $V(\mathscr{D}(X))=X$, which preserves the adjacency. This implies that $\overrightarrow{\mathcal{G}} \cong \mathscr{D}(X)$. Hence, $\mathcal{G} \cong \mathrm{D}(X)$. A simple example is shown in Figure 1.

Conversely, suppose that $\mathcal{G} \cong \mathrm{D}(X)$ for a finite set of positive integers $X$. It is obvious that $\mathcal{G}$ is a comparability graph.

If $\mathcal{G}$ is a $Y, Z$-bigraph, then directing every edge from $Y$ to $Z$ yields a transitive orientation. Hence, we have the following corollary. 


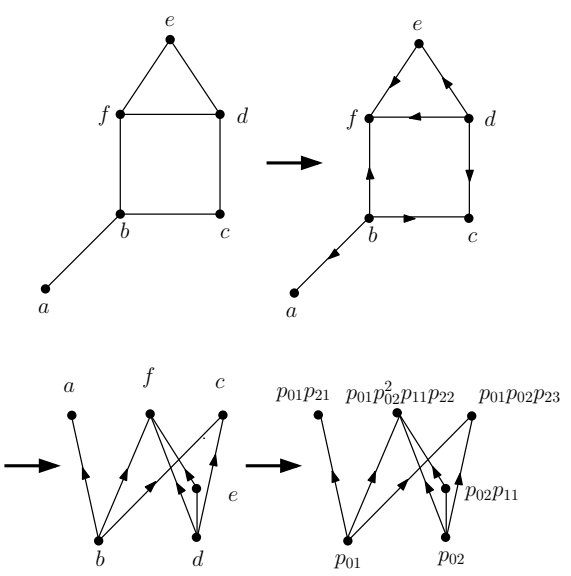

FiguRE 1. Example of comparable $\mathcal{G}$ for Theorem 2.1 .

Corollary 2.2. For a connected $Y, Z$-bigraph $\mathcal{B}$, there exists a finite set of positive integers $X$ such that $\mathcal{B} \cong \mathrm{D}(X)$.

Corollary 2.3. There exists $X \subseteq \mathbb{N}$ such that $\mathrm{D}(X) \cong C_{n}$ if and only if $n$ is even or $n=3$.

Proof. First, suppose that $C_{n} \cong \mathrm{D}(X)$ for $X \subseteq \mathbb{N}$. Then, by Theorem 2.1, there is a transitive orientation on $C_{n}$. Let $v_{0} \in V\left(\overrightarrow{C_{n}}\right)$ be a source. Then, $d^{-}(w) \geq 1$ for every $w \in N\left(v_{0}\right)$. Consider the following cases.

Case (i). There is a $w \in N\left(v_{0}\right)$ such that $d^{+}(w)>0$. In this case, there is a vertex $w^{\prime} \in V\left(\overrightarrow{C_{n}}\right)$ such that $\left(w, w^{\prime}\right) \in E\left(\overrightarrow{C_{n}}\right)$ where $w \in N\left(v_{0}\right)$. Since $C_{n}$ is a comparability graph, then $\left(v, w^{\prime}\right) \in E\left(\overrightarrow{C_{n}}\right)$. So $\overrightarrow{C_{n}}$ contains the triangle $v_{0} w w^{\prime}$. Therefore, in this case, $n=3$.

Case (ii). $d^{+}(w)=0$ for all $w \in N\left(v_{0}\right)$. Suppose that $N\left(v_{0}\right)=$ $\left\{v_{11}, v_{12}\right\}$. So $v_{11}$ and $v_{12}$ are sinks. For $i \geq 1$, let $N\left(v_{i k}\right)=\left\{v_{(i-1) k}\right.$, $\left.v_{(i+1) k}\right\}$ where $v_{01}=v_{02}=v_{0}$. Since $C_{n}$ is a comparability graph, $v_{i 1}$ and $v_{i 2}$ are sinks if $i$ is odd; otherwise, each is a source. Hence, $v_{i 1}$ and $v_{i 2}$ are not adjacent. Now, finiteness of $\overrightarrow{C_{n}}$ yields that there is an $i_{0}$ such that $v_{i_{0} 1}=v_{i_{0} 2}$, and therefore, $\left|V\left(\overrightarrow{C_{n}}\right)\right|$ is even. 
Conversely, suppose that $n$ is even. Since $C_{n}$ is a bigraph, by Corollary $2.2, C_{n}$ is a comparability graph, and by Theorem 2.1 , there is an $X \subseteq \mathbb{N}$ such that $C_{n} \cong \mathrm{D}(X)$. For $n=3$, let $X=\{a, a b, a b c\}$ for arbitrary $a, b, c \in \mathbb{N}^{*}$. It is easy to see that $\mathrm{D}(X) \cong C_{3}$.

If $\mathrm{D}(X)$ contains a cycle of odd length, say $n$, by the same argument as in the proof of Corollary 2.3, it is found that $\mathrm{D}(X)$ contains a triangle. So, we have the following corollary.

Corollary 2.4. The graph $\mathrm{D}(X)$ is bipartite if and only if it does not contain a triangle.

3. Relating the graphs of $\Delta(X), \Gamma(X), B(X)$ and $\mathrm{D}(X)$. In this section, we study certain parameters of a divisibility graph in comparison to the three graphs $\Gamma(X), \Delta(X)$ and $B(X)$. We also find a relation between the structure of $\mathrm{D}(X)$ and the other graphs.

3.1. Distance, diameter, girth and the number of components. Throughout this subsection, $X$ is a finite subset of $\mathbb{N}$ and $X \neq\{1\}$. Since $V(\mathrm{D}(X))=V(\Gamma(X))$ and $\{a, b\} \in E(\mathrm{D}(X))$ yield $\{a, b\} \in$ $E(\Gamma(X))$, we obtain that $\mathrm{D}(X)$ is a subgraph of $\Gamma(X)$. This is a key component for studying the parameters of $\mathrm{D}(X)$.

Note that this relation does not imply that all parameters of these graphs are the same. To show this, for every $n \in \mathbb{N}^{*}$, suppose that $p_{1}, \ldots, p_{n}$ are distinct primes. Let

$$
X=\left\{p_{i} \prod_{j=1}^{n} p_{j}, 1 \leq i \leq n\right\} .
$$

It is easy to see that $\Gamma(X)=\Delta(X)=K_{n}, B(X)=K_{n, n}$, and $\mathrm{D}(X)$ consists of $n$ isolated vertices.

In the following example, we show that it is possible to find sets $X$ and $Y$ such that $\mathrm{D}(X) \cong \mathrm{D}(Y)$, while $\Gamma(X) \varsubsetneqq \Gamma(Y)$. We also find $X$ and $Y$ such that $\mathrm{D}(X) \varsubsetneqq \mathrm{D}(Y)$, while $\Gamma(X) \cong \Gamma(Y)$.

Example 3.1. If $X=\{24,36\}$ and $Y=\{2,3\}$, then $\mathrm{D}(X) \cong \mathrm{D}(Y) \cong$ $2 K_{1}$. However, $\Gamma(X) \cong K_{2}$ and $\Gamma(Y) \cong 2 K_{1}$. If $X=\{24,36\}$ 
and $Y=\{2,12\}$, then $\mathrm{D}(X) \cong 2 K_{1}$ and $\mathrm{D}(Y) \cong K_{2}$. However, $\Gamma(Y) \cong \Gamma(Y) \cong K_{2}$.

Lemma 3.2. Some relations among $\mathrm{D}(X), \Gamma(X)$ and $\Delta(X)$ are as follows:

(i) $n(\mathrm{D}(X)) \geq n(\Gamma(X))=n(\Delta(X))=n(B(X))$.

(ii) If $[x]_{\mathrm{D}(X)}=[y]_{\mathrm{D}(X)}$, then $d_{\mathrm{D}(X)}(x, y) \geq d_{\Gamma(X)}(x, y)$.

(iii) $\operatorname{diam}(\mathrm{D}(X)) \geq \operatorname{diam}(\Gamma(X))$.

(iv) If $\mathrm{g}(\mathrm{D}(X)) \neq 0$, then $\mathrm{g}(\mathrm{D}(X)) \geq \mathrm{g}(\Gamma(X))$.

(v) If $\mathrm{g}(\mathrm{D}(X)) \neq 3$, then $\mathrm{g}(\mathrm{D}(X))$ is even.

(vi) For every $n \in \mathbb{N}$, there is a set $X$ such that $\mathrm{D}(X) \cong P_{n}$.

Proof. Part (i) is an immediate consequence of [5, Lemma 1 (c)] and the fact that $E(\mathrm{D}(X)) \subseteq E(\Gamma(X))$. The proofs of (ii)-(iv) are straightforward. Parts (v) and (vi) are obtained by Corollaries 2.3 and 2.2 , respectively.

In Table 1, we show that all possibilities given by Lemma 3.2 (i)-(iv) can arise.

TABLE 1. Examples of all possibilities for Lemma 3.2.

\begin{tabular}{|c|c|c|}
\hline$X$ & $\Gamma(X)$ (left), $\mathrm{D}(X)$ (right) & Parameters \\
\hline$\{2,6,12,14,15\}$ & &
\end{tabular}


3.2. Subgraphs of $\mathrm{D}(X)$ and $B(X)$. Corollary 2.2 asserts that, if $Y$ is a finite set of positive integers, then there exists a finite set of positive integers $X$, such that $B(Y) \cong \mathrm{D}(X)$. So, the structures of $B(X)$ and $\mathrm{D}(X)$ may be comparable.

\section{Lemma 3.3.}

(i) Suppose that $Y \neq\{1\}$ is a non-empty subset of $\mathbb{N}$. If $B(Y) \cong$ $\mathrm{D}(X)$ for some nonempty subset $X \neq\{1\}$ of $\mathbb{N}$, then there exist two subsets $X_{1}$ and $X_{2}$ such that $\mathrm{D}\left(X_{i}\right)$ is a null graph for $i=1,2 ;\left|X_{1}\right|=$ $|Y|,\left|X_{2}\right|=|\rho(Y)|$ and $X=X_{1} \cup X_{2}$.

(ii) If $X=X_{1} \cup X_{2}$ such that $X_{1} \cap X_{2}=\emptyset$ and $\mathrm{D}\left(X_{i}\right)$ is a null graph for $i=1,2$, then there is a $Y$ with $\mathrm{D}(X) \cong B(Y)$.

Proof.

(i) Since $B(Y) \cong \mathrm{D}(X)$, there is a bijection $\theta: V(B(Y)) \rightarrow$ $V(D(X))$. Now, since $B(Y)$ is a $Y, \rho(Y)$-bigraph, $\mathrm{D}(X)$ is also a $\theta(Y), \theta(\rho(Y))$-bigraph. Let $X_{1}=\theta(Y)$ and $X_{2}=\theta(\rho(Y))$. Then, since $\mathrm{D}(X)$ is bipartite, no two elements of $X_{1}$ and $X_{2}$ divide each other.

(ii) By hypothesis, it is easy to see that $\mathrm{D}(X)$ is a bigraph. So, from [5, Theorem 1], the proof is complete.

Suppose $X$ is a fixed finite subset of positive integers. The following theorem describes a relation between the subgraph structures of $\mathrm{D}(X)$ and $B(X)$.

Theorem 3.4. If $\operatorname{deg}(v) \leq 2$ for every vertex $v$ of $B(X)$, then $\mathrm{D}(X)$ is acyclic.

Proof. If $\operatorname{deg}(v) \leq 2$ for every vertex $v$ of $B(X)$, then every connected component of $B(X)$ is a path or a cycle of length $n$ where $n$ is even. Let $B_{1}$ be a connected component of $B(X)$. If $B_{1}$ is $C_{4}$ or a path, then by [5, Theorem 3], the corresponding connected component of $\Gamma(X)$ is acyclic, and hence, the corresponding connected component of $\mathrm{D}(X)$ is acyclic. Suppose that

$$
B_{1}=\left(p_{1}, x_{1}, p_{2}, x_{2}, \ldots, p_{k}, x_{k}, p_{1}\right)
$$


is a cycle of length $2 k$ where $k \geq 3$, and $\mathrm{D}_{1}$ is the connected component of $\mathrm{D}(X)$ corresponding to $B_{1}$. Obviously, $V\left(\mathrm{D}_{1}\right)=\left\{x_{1}, x_{2}, \ldots, x_{k}\right\}$. Since, for each $1 \leq i \leq k$, there is an element in $\rho\left(x_{i}\right) \backslash \rho\left(x_{j}\right)$ where $j \neq i$, the set $\left\{x_{1}, x_{2}, \ldots, x_{k}\right\}$ is an independent set in $\mathrm{D}(X)$.

Now, suppose $\mathrm{D}(X)$ contains an induced subgraph $\mathrm{D}^{\prime}$ which is isomorphic to the complete graph $K_{n}, n \geq 3$. Comparability asserts that this subgraph contains a source. If we omit this source from the vertex set of $\mathrm{D}^{\prime}$, we obtain another induced subgraph isomorphic to $K_{n-1}$, which has a source as well. By repetition of this method, we conclude that the vertex set of $\mathrm{D}(X)$ is the set $\left\{v_{1}, \ldots, v_{n}\right\}$, where $v_{i}$ divides $v_{j}$ for every $1 \leq i \leq j \leq n$. If $p$ is a prime divisor of $v_{1}$, then $p$ divides each $v_{i}, 2 \leq i \leq n$. Thus, the set $\left\{p, v_{1}, v_{2}, \ldots, v_{n}\right\}$ induces a subgraph $K_{1, n}$ in $B(X)$. Specifically, every triangle in $\mathrm{D}(X)$ creates an induced subgraph $K_{1,3}$ in $B(X)$. Since $\mathrm{D}(X) \subseteq \Gamma(X)$, by [5, Theorem 2], we conclude that $B(X)$ contains $C_{6}$ or $K_{1,3}$ as an induced subgraph. In fact, if $B(X)$ contains $C_{6}$ as an induced subgraph, then the corresponding subgraph of $\mathrm{D}(X)$ is acyclic. We will prove this fact in Theorem 3.4. We obtain the following corollary.

Corollary 3.5. If $\mathrm{D}(X)$ contains a triangle, then $B(X)$ contains $K_{1,3}$ as an induced subgraph.

It is easy to see that, for $X=\{2,6,10\}$, the converses of Theorem 3.4 and Corollary 3.5 are valid.

4. Divisibility graph for the product of two sets. In this section, we study the divisibility graph for the product of two nonempty subsets of positive integers. Throughout this section, $X$ and $Y$ are two non-empty subsets of positive integers.

The set $\{x y ; x \in X, y \in Y\}$ is denoted by $X Y$. The Cartesian product of $X$ is $Y, X \times Y=\{(x, y) ; x \in X, y \in Y\}$.

The strong product $\mathcal{G}_{1} \otimes \mathcal{G}_{2}$ of two graphs $\mathcal{G}_{1}$ and $\mathcal{G}_{2}$ is a graph with vertex set $\left\{(x, y) ; x \in V\left(\mathcal{G}_{1}\right), y \in V\left(\mathcal{G}_{2}\right)\right\}$, and the two vertices $\left(x_{1}, y_{1}\right)$ and $\left(x_{2}, y_{2}\right)$ are adjacent if one of the following conditions occurs $[\mathbf{3}$, Chapter 5].

(1) $x_{1} x_{2} \in E\left(\mathcal{G}_{1}\right)$ and $y_{1} y_{2} \in E\left(\mathcal{G}_{2}\right)$; 
(2) $x_{1}=x_{2}, y_{1} y_{2} \in E\left(\mathcal{G}_{2}\right)$;

(3) $x_{1} x_{2} \in E\left(\mathcal{G}_{1}\right), y_{1}=y_{2}$.

Where $u$ and $v$ are vertices of a graph, we denote $\{u, v\}((u, v))$, for $u v$, where the graph is not directed (is directed).

Now, let $\sim$ be the equivalence relation on $X \times Y$ such that:

$$
(x, y) \sim\left(x^{\prime}, y^{\prime}\right) \Longleftrightarrow x y=x^{\prime} y^{\prime}
$$

Definition 4.1. For two non-empty sets $X$ and $Y$, a graph with vertex set $\{[(x, y)] ;(x, y) \in X \times Y\}$ is denoted by $\mathrm{D}((X \times Y) / \sim)$, where $[(x, y)]$ is the equivalence class with respect to relation $\sim$, containing $(x, y)$. Two equivalence classes $[(x, y)]$ and $\left[\left(x^{\prime}, y^{\prime}\right)\right]$ are adjacent in $\mathrm{D}((X \times Y) / \sim)$ if and only if there are $\left(x_{1}, y_{1}\right) \in[(x, y)]$ and $\left(x_{2}, y_{2}\right) \in\left[\left(x^{\prime}, y^{\prime}\right)\right]$ such that they are adjacent in the underlying graph of $\mathscr{D}(X) \otimes \mathscr{D}(Y)$.

For simplicity, whenever the sets $X$ and $Y$ are clear from the context, we denote the graphs $\mathrm{D}((X \times Y) / \sim), \mathrm{D}(X Y)$ and the underlying graph of $\mathscr{D}(X) \otimes \mathscr{D}(Y)$ by $\mathrm{D}_{\sim}, \mathrm{D}$ and $\mathrm{D}_{\otimes}$, respectively.

By the definitions of these graphs, it is obvious that

$$
\left|V\left(\mathrm{D}_{\otimes}\right)\right| \geq\left|V\left(\mathrm{D}_{\sim}\right)\right|=|V(\mathrm{D})| \text {. }
$$

Also, $\mathrm{D}_{\sim} \cong \mathrm{D}_{\bigotimes}$ if and only if every equivalence class of $\sim$ contains only one element.

We are interested in finding the relations among these three graphs.

Theorem 4.2. For two arbitrary sets $X, Y \subseteq \mathbb{N}$ :

(i) If $\mathrm{D} \cong \mathrm{D}_{凶}$, then all three graphs are isomorphic.

(ii) $\mathrm{D}_{\sim}$ is isomorphic to a subgraph of $\mathrm{D}$. Also, if $\rho(X) \cap \rho(Y)=\emptyset$, then $\mathrm{D}_{\sim} \cong \mathrm{D} \cong \mathrm{D}_{\otimes}$.

(iii) For each $K \subseteq\left\{\mathrm{D}_{\sim}, \mathrm{D}_{\square}, \mathrm{D}\right\}$, where $|K| \neq 1$ and $K \neq\left\{\mathrm{D}_{\square}, \mathrm{D}\right\}$, there exist $X$ and $Y$ such that the elements of $K$ are isomorphic and the others are not. 
Proof.

(i) Note that $\mathrm{D} \cong \mathrm{D}_{\otimes}$ yields:

$$
\left|V\left(\mathrm{D}_{\otimes}\right)\right|=\left|V\left(\mathrm{D}_{\sim}\right)\right|=|V(\mathrm{D})| \text {. }
$$

This means that every equivalence class of $\sim$ contains only one element. So, we have

$$
\left\{[(x, y)],\left[\left(x^{\prime}, y^{\prime}\right)\right]\right\} \in E\left(\mathrm{D}_{\sim}\right)
$$

if and only if $\left\{(x, y),\left(x^{\prime}, y^{\prime}\right)\right\} \in E\left(\mathrm{D}_{\otimes}\right)$. Without loss of generality, assume there is a directed edge from $(x, y)$ to $\left(x^{\prime}, y^{\prime}\right)$ in $\mathscr{D}(X) \otimes \mathscr{D}(Y)$. By the definition of the strong product of two graphs, one may easily obtain that $x y$ divides $x^{\prime} y^{\prime}$. So, $\left\{x y, x^{\prime} y^{\prime}\right\} \in E(\mathrm{D})$.

Therefore, the maps

$$
f_{1}: V\left(\mathrm{D}_{\otimes}\right) \longrightarrow V\left(\mathrm{D}_{\sim}\right) \quad \text { and } \quad f_{2}: V\left(\mathrm{D}_{\sim}\right) \longrightarrow V(\mathrm{D})
$$

with the properties $f_{1}((x, y))=[(x, y)]$ and $f_{2}((x, y))=x y$, respectively, are bijections which preserve the adjacency. Hence, $\mathrm{D}_{\sim} \cong \mathrm{D} \cong$ $\mathrm{D}_{凶}$

(ii) We show that, for arbitrary sets $X$ and $Y, \mathrm{D}_{\sim}$ is isomorphic to a subgraph of $\mathrm{D}$. We define the map $f: V\left(\mathrm{D}_{\sim}\right) \rightarrow V(\mathrm{D})$ by $f([(x, y)])=x y$. From the definition of $\sim$, it is clear that $f$ is well defined.

Let $[(x, y)]$ and $\left[\left(x^{\prime}, y^{\prime}\right)\right]$ be two adjacent vertices of $\mathrm{D}_{\sim}$. Then, by Definition 4.1 , there are vertices,

$$
\left(x_{1}, y_{1}\right) \in[(x, y)] \text { and }\left(x_{2}, y_{2}\right) \in\left[\left(x^{\prime}, y^{\prime}\right)\right]
$$

which are adjacent in $\mathscr{D}(X) \otimes \mathscr{D}(Y)$. From the definition of $\sim$, one may easily obtain that $x_{1} y_{1}$ divides $x_{2} y_{2}$, or $x_{2} y_{2}$ divides $x_{1} y_{1}$. So, we have:

$$
\begin{aligned}
\left\{[(x, y)],\left[\left(x^{\prime}, y^{\prime}\right)\right]\right\} \in E\left(\mathrm{D}_{\sim}\right) & \Longrightarrow\left\{\left(x_{1}, y_{1}\right),\left(x_{2}, y_{2}\right)\right\} \in E(\mathscr{D}(X) \otimes \mathscr{D}(Y)) \\
& \Longrightarrow x_{1} y_{1} \mid x_{2} y_{2} \quad \text { or } \quad x_{2} y_{2} \mid x_{1} y_{1} \\
& \Longrightarrow\left\{x_{1} y_{1}, x_{2} y_{2}\right\} \in E(\mathrm{D}) \\
& \Longrightarrow\left\{f\left(\left[\left(x_{1}, y_{1}\right)\right]\right), f\left(\left[\left(x_{2}, y_{2}\right)\right]\right)\right\} \in E(\mathrm{D}) \\
& \Longrightarrow\left\{f([(x, y)]), f\left(\left[\left(x^{\prime}, y^{\prime}\right)\right]\right) \in E(\mathrm{D})\right\} .
\end{aligned}
$$

Thus, $f$ imbeds $\mathrm{D}_{\sim}$ to $\mathrm{D}$. 


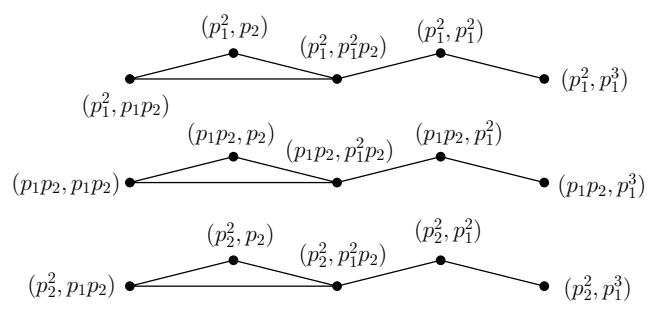

$\mathrm{D}_{\square}$

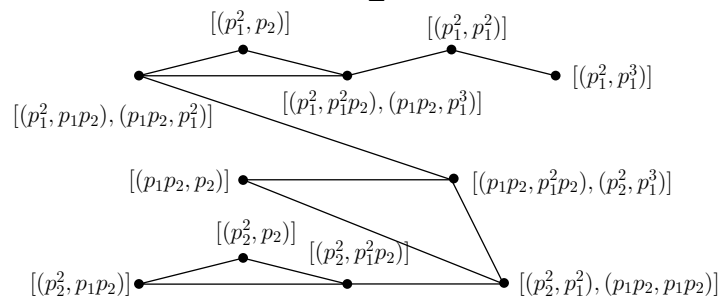

$\mathrm{D} \sim$

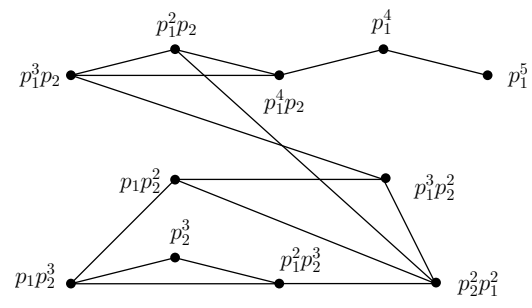

D

FIGURE 2. Illustration of proof of Theorem 4.2 (iii).

Now, suppose $\rho(X) \cap \rho(Y)=\emptyset$. Suppose $\left(x_{1}, y_{1}\right) \sim\left(x_{2}, y_{2}\right)$ where $x_{1}, x_{2} \in X$ and $y_{1}, y_{2} \in Y$. Since $\rho(X) \cap \rho(Y)=\emptyset$, we obtain $x_{1}=x_{2}$ and $y_{1}=y_{2}$. This means that every equivalence class is of order 1 and $\mathrm{D}_{\sim} \cong \mathrm{D}_{\square}$. Also, $\rho(X) \cap \rho(Y)=\emptyset$ includes the following:

$$
\begin{aligned}
\left\{x y, x^{\prime} y^{\prime}\right\} \in E(\mathrm{D}) & \Longleftrightarrow x y \mid x^{\prime} y^{\prime} \text { or } x^{\prime} y^{\prime} \mid x y \\
& \Longleftrightarrow x\left|x^{\prime}, y\right| y^{\prime} \text { or } x^{\prime}\left|x, y^{\prime}\right| y \\
& \Longleftrightarrow\left\{(x, y),\left(x^{\prime}, y^{\prime}\right)\right\} \in E\left(\mathrm{D}_{\square}\right) .
\end{aligned}
$$

Therefore, the proof of (ii) is complete. 
(iii) For a given $K \subseteq\left\{\mathrm{D}_{\sim}, \mathrm{D}_{\bigotimes}, \mathrm{D}\right\}$, we will find proper subsets $X$ and $Y$ of positive integers such that the elements of $K$ are isomorphic, but the other elements of $\left\{\mathrm{D}_{\sim}, \mathrm{D}_{\varpi}, \mathrm{D}\right\}$ are not isomorphic to elements of $K$.

Suppose $\left\{p_{1}, p_{2}, p_{3}\right\}$ is a set of distinct primes,

$$
X=\left\{p_{1}^{2}, p_{1} p_{2}, p_{2}^{3}\right\}
$$

and

$$
Y=\left\{p_{2}, p_{1} p_{2}, p_{1}^{2}, p_{1}^{3}, p_{1}^{2} p_{2}\right\} .
$$

In this case, $\mathrm{D}_{\bigotimes}$ is not connected. But, $\mathrm{D}$ and $\mathrm{D}_{\sim}$ are connected graphs with 11 vertices. So, they are not isomorphic to $\mathrm{D}_{\square}$. Also, $|E(\mathrm{D})|=\left|E\left(\mathrm{D}_{\sim}\right)\right|+2$. Hence, these two graphs are not isomorphic. The graphs $\mathrm{D}_{\sim}, \mathrm{D}_{\bowtie}$ and $\mathrm{D}$ are shown in Figure 2.

\begin{tabular}{|c|c|c|c|c|}
\hline$X$ & $Y$ & $\mathrm{D}_{\sim}$ & $\mathrm{D}_{\Downarrow}$ & $\mathrm{D}$ \\
\hline$\left\{p_{3}, p_{1} p_{3}\right\}$ & $\left\{p_{3}, p_{2} p_{3}\right\}$ & $\left.\left[\left(p_{1} p_{3}, p_{3}\right)\right]\left[\left(p_{1}, p_{3}\right)\right]\left[\left(p_{3}, p_{2}, p_{2} p_{3}\right)\right]\right]$ & $\left(p_{\left(p_{1} p_{3}, p_{3}\right)\left(p_{1} p_{3}, p_{2} p_{3}\right)}^{\left(p_{3}, p_{3}\right)}\right.$ & $\overbrace{p_{1} p_{3}^{2}}^{p_{p_{1}} p_{2} p_{3}^{2}}$ \\
\hline$\left\{p_{1}, p_{1} p_{2}\right\}$ & $\left\{p_{2}, p_{2}^{2}\right\}$ & $\overbrace{\left[\left(p_{1} p_{2}, p_{2}^{3}\right)\right]}^{\left[\left(p_{1}, p_{2}\right)\right]\left[\left(p_{1}, p_{2}^{3}\right)\right]}$ & $\overbrace{\left(p_{1} p_{2}, p_{2}\right)\left(p_{1} p_{2}, p_{2}^{3}\right)}^{\left(p_{1}, p_{2}\right)}$ & $p_{1} p_{2} \quad p_{1} p_{2}^{2}$ \\
\hline$\left\{p_{1} p_{2}, p_{1}^{2}\right\}$ & $\left\{p_{1} p_{2}, p_{2}^{3}\right\}$ & 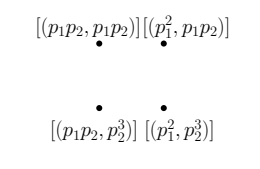 & $\begin{array}{c}\left(p_{1} p_{2}, p_{1} p_{2}\right)\left(p_{1}^{2}, p_{1} p_{2}\right) \\
\dot{\left(p_{1} p_{2}, p_{2}^{3}\right)}\left(\dot{p_{1}^{2}}, p_{2}^{3}\right)\end{array}$ & $\underbrace{p_{1}^{2} p_{2}^{2} p_{2}^{3}}_{p_{1} p_{2}^{4}}$ \\
\hline
\end{tabular}

TABLE 2. Examples of Theorem 4.2 (iii). 
For the other cases of $K$, we refer the reader to Table 2. In the first, second and third rows of Table $2, K$ is equal to $\left\{\mathrm{D}_{\sim}, \mathrm{D}_{\otimes}, \mathrm{D}\right\},\left\{\mathrm{D}_{\sim}, \mathrm{D}\right\}$ and $\left\{\mathrm{D}_{\sim}, \mathrm{D}_{\otimes}\right\}$, respectively.

In the following lemma, we find the structure of $\mathrm{D}(X)$ and $\mathrm{D}(Y)$ when $\mathrm{D}(X Y)$ is a complete graph.

Lemma 4.3. Suppose $X$ and $Y$ are two non-empty sets. If $\mathrm{D}(X Y)$ is complete, then both $\mathrm{D}(X)$ and $\mathrm{D}(Y)$ are complete.

Proof. Let $x, x^{\prime} \in X$ and $y \in Y$ be three arbitrary elements. We have $x y$ and $x^{\prime} y \in X Y$. Since $\mathrm{D}(X Y)$ is complete, then either $x y$ divides $x^{\prime} y$ or $x^{\prime} y$ divides $x y$. Hence, either $x$ divides $x^{\prime}$ or $x^{\prime}$ divides $x$. This means that $\mathrm{D}(X)$ is complete. The same argument shows that $\mathrm{D}(Y)$ is complete.

Lemma 4.4. Suppose $\mathrm{D}(X Y)$ is complete and $\rho(X) \cap \rho(Y)=\emptyset$. Then, $|X|=1$ or $|Y|=1$.

Proof. Since $\rho(X) \cap \rho(Y)=\emptyset$, by Theorem 4.2, $\mathrm{D}_{\square} \cong \mathrm{D}$. So $\mathrm{D}_{\square}$ is complete. Conversely, suppose there are $x_{1}, x_{2} \in X$ and $y_{1}, y_{2} \in Y$ such that $x_{1}<x_{2}$ and $y_{1}<y_{2}$. Since $\mathrm{D}_{\square}$ is complete,

$$
\left\{\left(x_{1}, y_{2}\right),\left(x_{2}, y_{1}\right)\right\} \in E\left(\mathrm{D}_{\otimes}\right) .
$$

By Definition 4.1, this is a contradiction.

Acknowledgments. The authors wish to thank Margaret Bayer and anonymous referees for helpful comments on a final version of the paper.

\section{REFERENCES}

1. A. Abdolghafourian and M.A. Iranmanesh, Divisibility graph for symmetric and alternating groups, Comm. Alg. 4 (2015), 2852-2862.

2. A.R. Camina and R.D. Camina, The influence of conjugacy class sizes on the structure of finite groups: A survey, Asian-Europ. J. Math. 4 (2011), 559-588.

3. W. Imrich and S. Klavzar, Product graphs, Wiley, New York, 2000.

4. M.A. Iranmanesh, Bipartite divisor graph, A survey, Electr. Notes Combin. 45 (2014), 43-49. 
5. M.A. Iranmanesh and Cheryl E. Praeger, Bipartite divisor graphs for integer subsets, Graphs Combin. 26 (2010), 95-105.

6. Mark L. Lewis, An overview of graphs associated with character degrees and conjugacy class sizes in finite groups, Rocky Mountain J. Math. 38 (2008), 175-211.

7. D.B. West, Introduction to graph theory 2, Prentice Hall, Upper Saddle River, NJ, 2001.

Department of Mathematics, Yazd University, Yazd, 89195-741, Iran Email address: a.abdolghafourian@stu.yazd.ac.ir

Department of Mathematics, Yazd University, Yazd, 89195-741, Iran Email address: iranmanesh@yazd.ac.ir 